\title{
DEFICIÊNCIA HÍDRICA NO CONSUMO DE ÁGUA DE DOIS CULTIVARES DE MILHO CRESCIDOS EM SOLUÇÃO NUTRITIVA (')
}

\author{
EDUARDO CARUSO MACHADO $\left({ }^{2,3}\right)$ e PEDRO ROBERTO FURLANI $\left({ }^{2,3}\right)$
}

\begin{abstract}
RESUMO
Estudaram-se no presente trabalho os efeitos da deficiência hídrica no crescimento e consumo de água de plantas dos cultivares de milho IAC-Maya e IACMaya latente crescidos em casa de vegetação e em solução nutritiva em recipientes cilíndricos de PVC (diâmetro $=15 \mathrm{~cm}$, altura $=100 \mathrm{~cm}$ ), com capacidade para 15 litros. A solução nutritiva, composta de (mg/litro): $\mathrm{Ca}(284), \mathrm{N}-\mathrm{NO}_{3}(297), \mathrm{N}^{-\mathrm{NH}_{4}}$ (37), K (140), P (6), Mg (41), Fe $(7,5), \mathrm{B}(0,5), \mathrm{Cu}(0,08), \mathrm{Mn}(1,0)$, Mo $(0,16) \mathrm{e}$ $\mathrm{Zn}(0,3)$, foi renovada a cada 30 dias. Nos estádios vegetativo e de florescimento, as plantas foram submetidas à deficiência hídrica através de a) adição de polietileno glicol e b) suspensāo da reposição da água evapotranspirada pelas plantas. O delineamento estatístico foi $o$ de blocos ao acaso com cinco repetições. A deficiência hídrica ocasionou, em ambos os cultivares, redução no consumo de água e na área foliar. Nos tratamentos onde a deficiência hídrica foi induzida pela suspensâo da reposição da água evapotranspirada, observou-se aumento das raízes de ambos os cultivares. Os resultados sugerem que a técnica de cultivo por hidroponia associada ao uso de agentes osmóticos e/ou suspensão da reposição da água evapotranspirada, pode ser utilizada em estudos de deficiência hídrica em plantas.
\end{abstract}

Termos de indexação: deficiência hídrica, agentes osmóticos, cultivo hidropônico.

(1) Apresentada na IX Reunião Latino-Americana de Fisiologia Vegetal, Viçosa (MG), julho de 1983. Recebida para publicação em 31 de maio de 1984.

(2) Seção de Fisiologia, Instituto Agronômico (IAC), Caixa Postal 28, 13100 - Campinas (SP).

(3) Bolsista do CNPq. 


\section{INTRODUCAXO}

A deficiência hídrica é uma das causas mais freqüentes de insucessos agrícolas, reduzindo acentuadamente a produção de grãos em milho. A irrigação pode corrigir tal problema: entretanto, em muitos casos, é inviável economicamente. Uma alternativa seria a utilização de cultivares co!n capacidade de resistir a períodos com deficiência hídrica sem grandes prejuízos à produção. MIRANDA (1972) relata que o complexo latência confere ao milho resistência ambiente, que é a capacidade dos indivíduos de sobreviver a ambientes desfavoráveis em umidade e calor. E importante, pois, que se compreendam e caracterizem os processos morfofisiológicos que possam conferir às plantas tal resistência, visando definir parâmetros a serem utilizados como auxiliares em programas de melhoramento de milho objetivando a seleção de plantas resistentes à seca.

Um dos principais problemas em estudo de relações hidricas em plantas diz respeito ao desenvolvimento de metodologia. $\mathrm{O}$ uso de agentes osmóticos em culturas hidropônicas tem sido utilizado com este objetivo (SULLIVAN et alii, 1978; BENNETT, 1979; SULLIVAN \& ROSS, 1979). O cultivo hidropônico oferece possibilidades de controle do consumo de água, avaliação do desenvolvimento do sistema radicular de plantas individuais e indução controlada de estresse hídrico. Destaca-se também a possibilidade de reproduzir condições semelhantes em ensaios conduzidos em diferentes épocas.

$O$ presente trabalho teve por objetivo avaliar os efeitos da deficiência hídrica no consumo de água por dois cultivares de milho, crescidos em solução nutritiva.

\section{MATERIAL E MÉTODOS}

Plantas homogêneas dos cultivares de milho IAC-Maya normal e IAC-Maya latente foram cultivadas sob condições não controladas de casa de vegetação, em recipientes cilíndricos de PVC $(100 \times 15 \mathrm{~cm}$ de diâmetro), contendo solução nutritiva composta de (mg/litro): Ca (284), $\mathrm{N}-\mathrm{NO}_{3}$ (297), $\mathrm{N}-\mathrm{NH}_{4}$ (37), K (140), P (6), Mg (41), Fe (7,5), B $(0,5), \mathrm{Cu}(0,08), \mathrm{Mn}(1,0)$, Mo $(0,16)$ e $\mathrm{Zn}(0,3)$. A solução nutritiva preparada com água de torneira foi renovada em intervalos de 30 dias.

A semeação foi feita $(6 / 10 / 82)$ em papel crepom Unitest embebido em água. Seis dias após a germinação $(15 / 10 / 82)$, as plântulas foram transferidas para recipientes retangulares (capacidade de 15 litros) com solução nutritiva, onde permaneceram até atingir $20 \mathrm{~cm}$ de altura $(25 / 10 / 82)$. Nesse 
estádio, as plantas foram selecionadas e transplantadas para os recipientes cilíndricos de PVC, onde permaneceram até o final do ensaio. Cada tubo de PVC continha uma única planta. As raízes foram continuamente supridas de oxigênio através de aeração com ar comprimido.

Os recipientes cilíndricos foram dispostos espaçados de $1 \mathrm{~m}$ entre linhas e $0,2 \mathrm{~m}$ entre plantas.

A indução de estresse hídrico foi feita de duas formas: primeira, diminuindo o potencial osmótico da solução nutritiva pela adição de polietileno glicol-600 (PEG-600). A suspensão do estresse foi feita pela troca da solução nutritiva com PEG-600 por outra normal. A relação entre o potencial osmótico da solução nutritiva em função do volume de PEG-600 adicionado foi determinada com osmômetro. A segunda forma consistiu na suspensão da reposição de água evapotranspirada pelas plantas. Neste caso, a quantidade de raízes imersas em solução decresce vagarosa e continuamente. A reposição de água foi iniciada quando as plantas apresentavam sintomas visíveis de deficiência hídrica, ou após um tempo predeterminado.

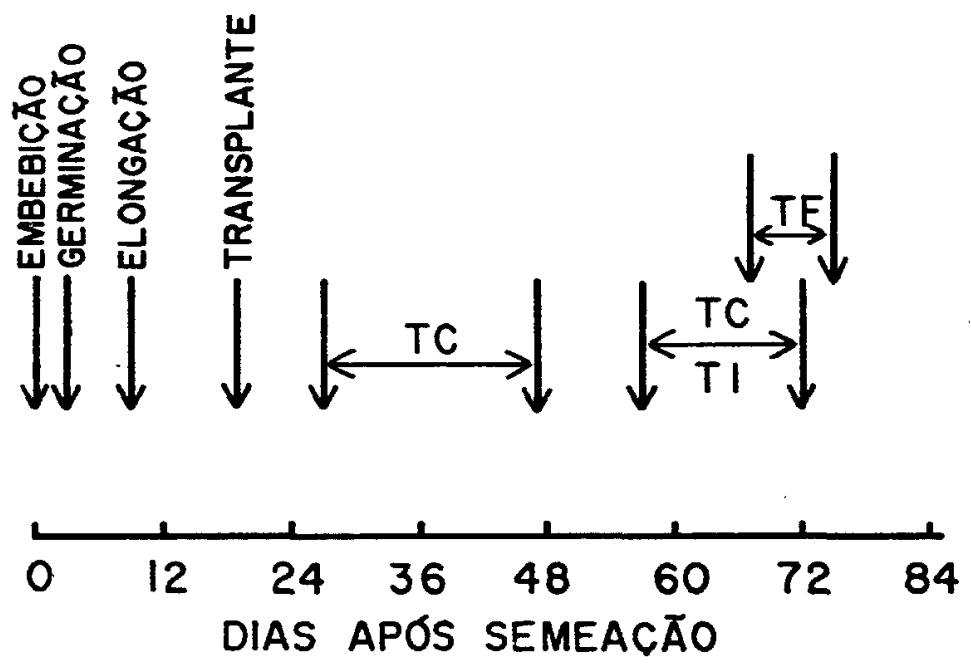

FIGURA 1 - Épocas e formas de aplicação de estresse hídrico em milho. TF: estresse de -7 bar durante a polinização; TI: estresse pela suspensão da reposição de água evapotranspirada, na polinização, e TC: estresse cíclico, com reposição de água quando nenessário. 
Os tratamentos foram os seguintes: TN: controle, no qual as plantas não sofreram estresse; TF: estresse de -7 bar (adição de 3 volumes, um por dia, de $400 \mathrm{ml}$ de PEG-600 em cada tubo) por um período de oito dias durante o florescimento; TI: estresse imposto por suspensão da reposição de água evapotranspirada por oito dias antes da emergência da inflorescência masculina, e TC: reposição da água evapotranspirada somente quando as plantas apresentavam sintomas visíveis de deficiência hídrica. Um esquema das épocas e formas de aplicação de estresse é apresentado na Figura 1 .

O experimento foi delineado em blocos ao acaso com cinco repetiços por tratamento.

Foram efetuadas as seguintes avaliações: (a) consumo de água (mililitro de água/planta) determinado por um sistema automático de reposição de água; $(b)$ área foliar, estimada a cada sete dias pela expressão comprimento da folha $x$ largura da folha x 0,75 (MONTGOMERY, 1911); (c) profundidade do sistema radicular.

\section{RESULTADOS E DISCUSSÃO}

$\mathrm{O}$ consumo de água ( $\left.\mathrm{ml} \mathrm{de} \mathrm{água.planta}{ }^{-1} \cdot \mathrm{dia}^{-1}\right)$ pelos cultivares IAC-Maya normal (normal) e IAC-Maya latente (latente) até o florescimento no tratamento TN é mostrado na Figura 2. Ambos apresentaram um comportamento semelhante; entretanto, o latente consumiu uma quantidade ligeiramente maior durante praticamente todo o periodo experimental. A Figura 3 mostra que a taxa de transpiração, isto é, perda de água por unidade de área foliar $\left(\mathrm{ml}\right.$ de água.dm $\left.{ }^{-2} \cdot \mathrm{dia}^{-1}\right)$, variou entre 5 e 42 . Essa variação está relacionada com a demanda evaporativa do ar. Observa-se que ambos os cultivares apresentaram, praticamente, a mesma taxa de transpiração quando não submetidos a estresse hídrico (TN). Entretanto, em condiçб̄es de maior demanda evaporativa do ar, o cultivar normal apresentou maiores taxas de transpiração que o latente. Tal comportamento parece estar relacionado com a maior sensibilidade para o fechamento de estômatos do segundo em relação ao normal (BRUNINI et alii, 1982a; 1982b; 1983).

No tratamento TC, as plantas foram submetidas a dois ciclos de estresse por suspensão da reposição da água evapotranspirada. No intervalo, foi instalado um sistema automático de reposição e controle de consumo de água. No primeiro ciclo de estresse, as plantas apresentaram sinais visíveis de deficiência hídrica somente quando cerca de 30\% das raízes (comprimento das raízes imersas/comprimento total das raízes $\times 100$ ) estavam imersas. Todavia, essa quantidade em termos de volume de raízes imersas/volume de 


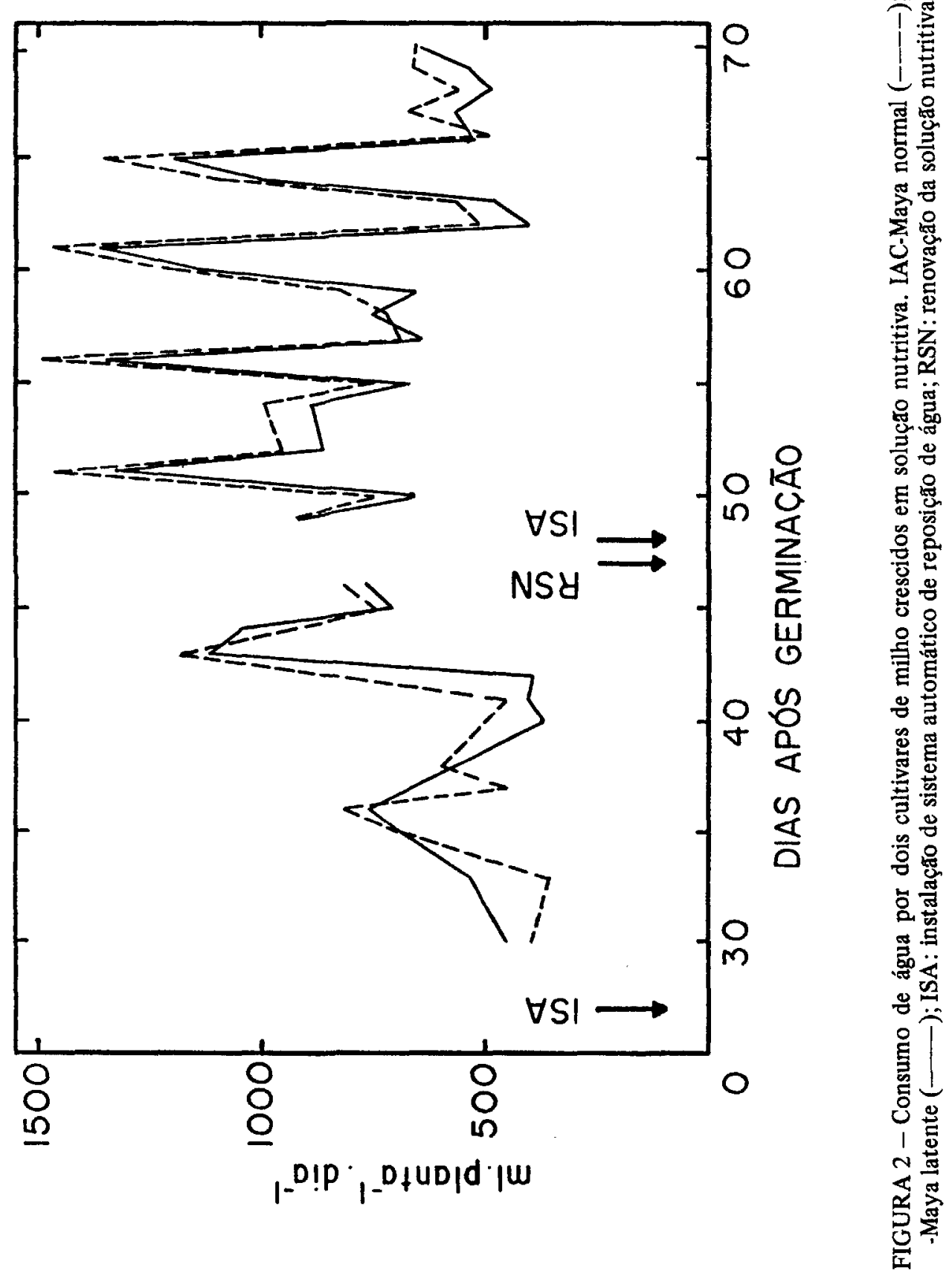




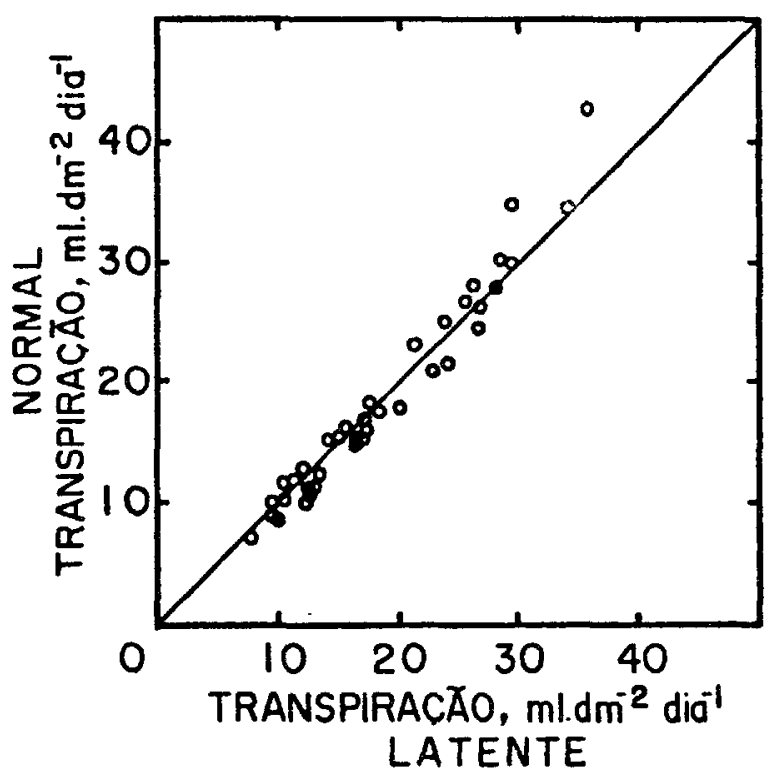

FIGURA 3 - Comparação entre as taxas de transpiração dos cultivares de milho IACMaya normal e IAC-Maya latente.

raizes total, foi muito menor, uma vez que somente algumas raízes que se elongaram mais rapidamente é que ficaram imersas na solução. O consumo de água no período de estresse foi 49\% (296ml.planta ${ }^{-1} \cdot \mathrm{dia}^{-1}$ ) e $58 \%$ (344m1. planta $\left.^{-1} \cdot \mathrm{dia}^{-1}\right)$ em relação à testemunha latente $\left(604 \mathrm{ml}\right.$.planta $\left.{ }^{-1} \cdot \mathrm{dia}^{-1}\right)$ e normal (595ml.planta ${ }^{-1}$. dia $^{-1}$ ) respectivamente. O consumo do cultivar normal foi ligeiramente maior nesse período de estresse, concordando com os resultados encontrados por BRUNINI et alii (1982a, 1982b, 1983) e CAMARGO et alii (1982), sob condiçбes de campo.

O controle do consumo de água após o primeiro ciclo revelou que as plantas que sofreram estresse não conseguiram recuperar a taxa de transpiração no mesmo nível das testemunhas, isto é, as taxas de transpiração das plantas estressadas foram $25 \%$ menores que as testemunhas. O consumo de água durante o período de recuperação, apresentado na Figura 4, sugere que as raizes que ficaram expostas ao ar não recuperaram sua capacidade plena de absorção. No segundo ciclo de estresse do tratamento TC, a taxa de transpiração média foi $66 \%\left(7,7 \mathrm{ml} \cdot \mathrm{dm}^{-2} \cdot \mathrm{dia}^{-1}\right)$ e $74 \%\left(8,9 \mathrm{ml} \cdot \mathrm{dm}^{-2} \cdot \mathrm{dia}^{-1}\right)$ em relação às testemunhas normal $\left(11,7 \mathrm{ml} \cdot \mathrm{dm}^{-2} \cdot \mathrm{dia}^{-1}\right)$ e latente $\left(12,1 \mathrm{ml} \cdot \mathrm{dm}^{-2} \cdot \mathrm{dia}^{-1}\right)$ respectivamente (Quadro 1). Após o segundo ciclo de estresse a reposição de água foi feita normalmente, isto é, não houve mais estresse. 
QUADRO 1 - Taxa de transpiração média em dois cultivares de milho

\begin{tabular}{lcc}
\hline & \multicolumn{2}{c}{ Cultivar } \\
\cline { 2 - 3 } Tratamento $\left({ }^{1}\right)$ & IAC-Maya normal & IAC-Maya latente \\
\cline { 2 - 3 } & 11,7 & 12,1 \\
TN & 8,4 & $\mathrm{dm}^{-2} \cdot \mathrm{dia}^{-1}$ \\
TF & 7,4 & 8,3 \\
TI & 7,7 & 7,2 \\
TC & 8,9 \\
\hline
\end{tabular}

( $\left.{ }^{1}\right)$ TN: testemunha; TF: estresse de -7 bar durante a polinização; TI: estresse pela suspensão da reposição de água evapotranspirada, na polinização, e TC: estresse cíclico, com reposiçāo de água quando necessário.

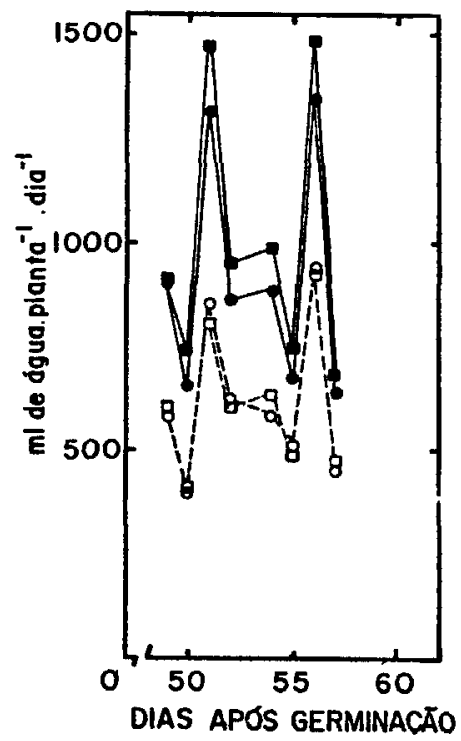

FIGURA 4 - Consumo de água por dois cultivares de milho após um período de estresse

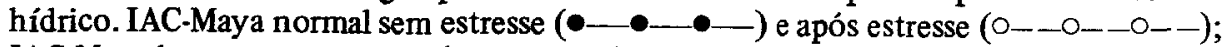
IAC-Maya latente'sem estresse (- - - - ) e após estresse (-- $\left.-\square_{-}--\right)$. 
No tratamento TI, a reposição de água foi suspensa por quinze dias, ou seja, sete dias antes do período de polinização. Assim, em oito dias durante a polinização, somente parte das raízes ficaram imersas em solução nutritiva. A taxa de transpiração média das plantas estressadas foi $63 \%(7,4 \mathrm{ml}$. $\left.\mathrm{dm}^{-2} \cdot \mathrm{dia}^{-1}\right)$ e $60 \%\left(7,2 \mathrm{ml} \cdot \mathrm{dm}^{-2} \cdot \mathrm{dia}^{-1}\right)$ em relaçđo às testemunhas normal $\left(11,7 \mathrm{ml} \cdot \mathrm{dm}^{-2} \cdot \mathrm{dia}^{-1}\right)$ e latente $\left(12,1 \mathrm{ml} \cdot \mathrm{dm}^{-2} \cdot \mathrm{dia}^{-1}\right)$ respectivamente (Quadro 1).

Nas plantas do tratamento TF, foi mantido o potencial de -7 bar por oito dias durante o período de polinização. A taxa de transpiração média

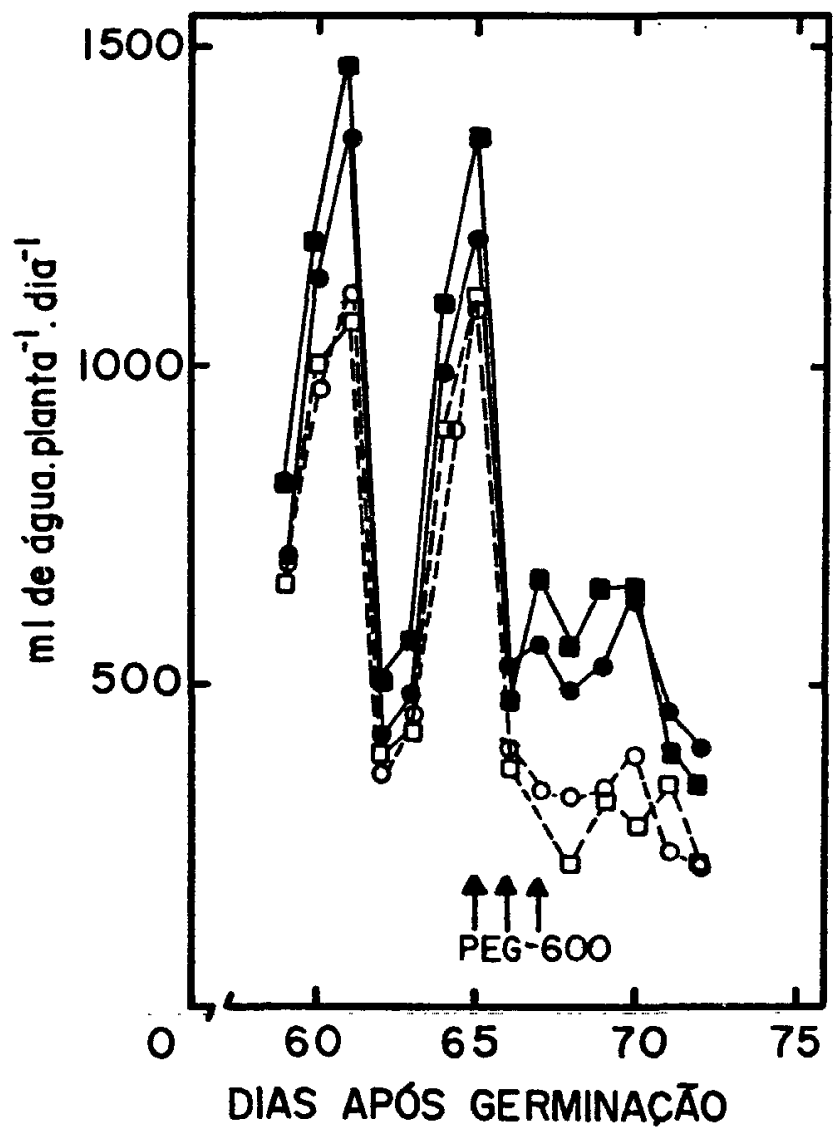

FIGURA 5 - Consumo de água por dois cultivares de milho com e sem estresse hídrico de -7 bar (adição de PEG-600). IAC-Maya normal sem estresse (๑-—-) e com

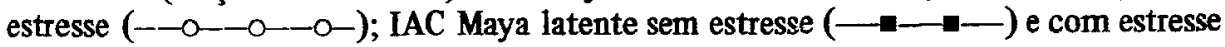
(- - - - - - - $)$. 
das plantas que sofreram estresse foi $70 \%\left(8,4 \mathrm{ml} \cdot \mathrm{dm}^{-2} \cdot \mathrm{dia}^{-1}\right)$ e $69 \%(8,3 \mathrm{ml}$. $\left.\mathrm{dm}^{-2} \cdot \mathrm{dia}^{-1}\right)$ em relação às testemunhas normal $\left(11,7 \mathrm{ml} \cdot \mathrm{dm}^{-2} \cdot \mathrm{dia}^{-1}\right)$ e latente $\left(12,1 \mathrm{ml} \cdot \mathrm{dm}^{-2} \cdot \mathrm{dia}^{-1}\right)$ respectivamente (Quadro 1$)$. O consumo diário de água, antes e durante o tratamento TI, é apresentado na Figura 5, onde fica evidenciada a queda no consumo apos a adição de PEG-600 à solução. Durante esse tratamento, o tempo permaneceu nublado, de forma que a demanda evaporativa foi baixa, o que poderia explicar a razão da queda acentuada do consumo de água pelas plantas testemunhas.

A figura 6 ilustra o desenvolvimento da área foliar das plantas testemunhas e daquelas que sofreram estresse pelo tratamento TC. Observa-se que as testemunhas normal e latente comportaram-se de forma semelhante. Ambos os cultivares sob estresse tiveram o desenvolvimento da área foliar prejudicado. Por ocasião do término do experimento (início da fase de enchimento de grãos), os comprimentos das raizes das plantas submetidas aos tratamentos TI e TC foram maiores que os das plantas dos tratamentos TF e TN (Quadro 2). Isso mostra que houve uma tendencia de as raízes crescerem em busca de água e nutrientes, o que foi observado também em sorgo por SULLIVAN et alii (1978).

QUADRO 2 - Efeitos da indução de estresse hídrico no crescimento da raiz de dois cultivares de milho. Resultados expressos em porcentagem em relação ao tratamento testemunha (TN)

\begin{tabular}{lll}
\hline & \multicolumn{2}{c}{ Cultivar } \\
\cline { 2 - 3 } Tratamento $\left({ }^{1}\right)$ & \multicolumn{1}{c}{$\begin{array}{c}\text { IAC-Maya } \\
\text { normal }\end{array}$} & $\begin{array}{c}\text { IAC-Maya } \\
\text { latente }\end{array}$ \\
\hline & \multicolumn{2}{c}{ Comprimento da raiz (\%) } \\
TN & $100(56)\left({ }^{2}\right)$ & $100(57)\left({ }^{2}\right)$ \\
TI & 145 & 137 \\
TC & 153 & 155 \\
& 100 & 10 \\
\hline
\end{tabular}

( $\left.{ }^{1}\right)$ TN: testemunha; TF: estresse de -7 bar durante a polinização; TI: estresse pela suspensão de reposição de água evapotranspirada, na polinização, e TC: estresse cíclico, com reposição de água quando necessário. $\left({ }^{2}\right)$ Comprimento médio das raízes do tratamento TN em cm/planta. 


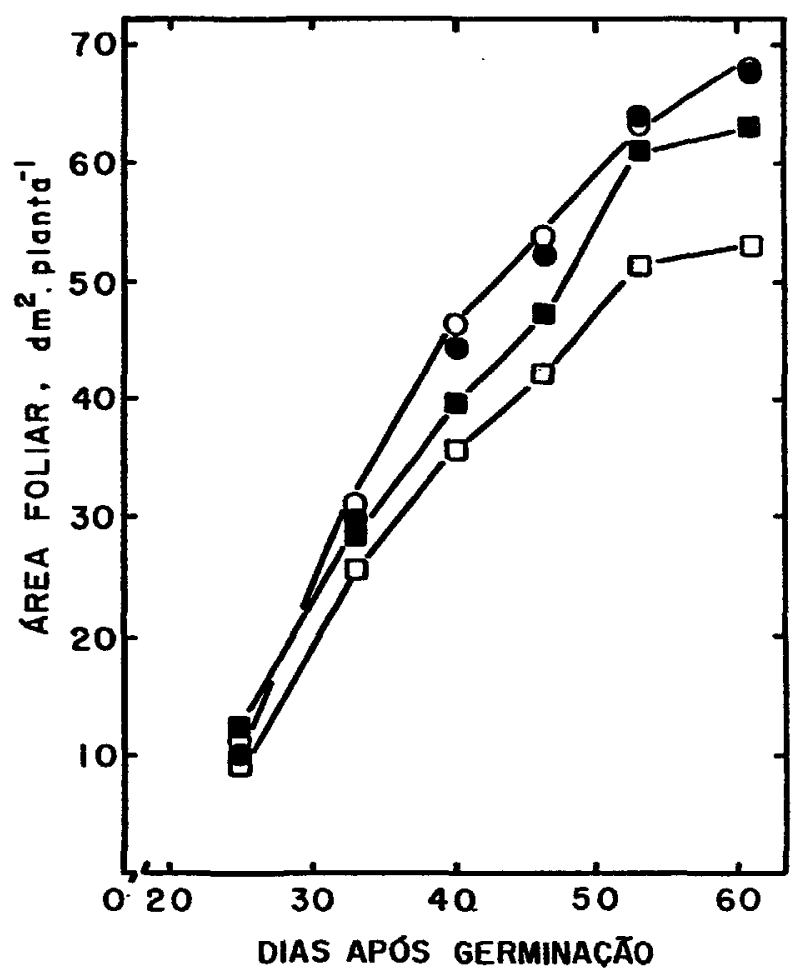

FIGURA 6 - Desenvolvimento da área foliar em dois cultivares de milho. IAC-Maya normal sem estresse ( $\longrightarrow \longrightarrow-$ ) e com estresse ( $\longrightarrow-\longrightarrow$ ); IAC-Maya latente sem estresse $(-0-0-0-)$ e com estresse $(\square \square-\square-)$ ).

Os resultados obtidos sugerem que o sistema de cultivo de plantas em solução nutritiva para estudo dos efeitos da deficiência hídrica sobre o consumo e crescimento de plantas foi adequado. O uso de PEG-600 como agente osmótico possibilitou controlar a intensidade e o período de duração do estresse. $O$ sistema de suspensão da reposição da água transpirada também demonstrou ser satisfatório para simular a deficiência hídrica, além de possibilitar estudos comparativos da capacidade de crescimento do sistema radicular.

\section{AGRADECIMENTO}

Os autores expressam seu agradecimento ao Sr. Arlindo Salles Nogueira, funcionário da Seção de Fisiologia, IAC, pela colaboração na montagem e condução deste trabalho. 


\title{
SUMMARY
}

\section{WATER DEFICIT EFFECTS ON WATER USE OF TWO CORN CULTIVARS GROWN IN NUTRIENT SOLUTIONS}

\begin{abstract}
Two corn cultivars ("IAC-Maya" and "IAC-Maya latente") were grown in hydroponic culture, in 15 liters PVC cilindric pots, in a greenhouse. The nutrient solutions were replaced each $\mathbf{3 0}$ days period. The plants were subjected to water stress, either by addition of polyethylene glycol-600 to the nutrient solutions at the flowering period, or by no reposition of water transpired by the plants at the flowering and vegetative periods. The experimental design consisted of randomized complete blocks with 5 replications. Reductions in water consumption, and leaf area were observed in both genotypes. An increase in the root length was observed only in the treatments that water stress was imposed by no reposition of water transpired. The results suggested that hydroponic culture may be a suitable method for studying the effects of water deficit in plants.
\end{abstract}

Index terms: water deficits, osmotic agents, hydroponic culture.

\section{REFERÊNCIAS BIBLIOGRÁFICAS}

BENNETT, J.M. Responses of grain sorghum (Sorghum bicolor (L) Moench) to osmotic stress imposed at various growth stages. Lincoln, Nebraska, University of Nebraska, 1979. 202p. Tese. (Ph. D.)

BRUNINI, O.; BATAGLIA, O.C.; CIARELLI, D.M.; CAMARGO, M.B.P. de \& MIRANDA, L.T. Características de resistência à seca em variedades de milho. In: CONGRESSO BRASILEIRO DE AGROMETEOROLOGIA, 3., Campinas, SP, 1983. Resumos. p.42-43.

; CAMARGO, M.B.P. de; DONZELLI, J.L.; ANGELOCCI, L.R.; MAGALHÃES, H.H.S.; SAWASAKI, E.; MIRANDA, L.T., VOLPE, C.A. \& FISCH, G.F. Sensibilidade estomatal de cultivares de milho em condiçơs de campo. In: CONGRESSO NACIONAL DE MILHO E SORGO, 14., Florianópolis, SC, 1982a. Resumo. p.103.

; DONZELLI, J.L.; CAMARGO, M.B.P. de; CABRAL, O.M.R.; SAWASAKI, E.; MIRANDA, L.T.; VOLPE, C.A.; ANGELOCCI, L.R. \& FISCH, G.F. Balanço hidrico em condiçoes de campo para cultivares de milho. In: CONGRESSO DE MILHO E SORGO, 14., Florianópolis, SC, 1982b. Resumo. p.104.

CAMARGO, M.B.P. de; BRUNIINI, O.; MACHADO, E.C.; DONZELLI, J.L.; MIRANDA, L.T.; SAWASAKI, E.; ANGELOCCI, L.R.; VOLPE, C.A. \& JANUÁRIO, M. Eficiência do uso de água por cultivares de milho. In: CONGRESSO NACIONAL DE MILHO E SORGO, 14., Florianópolis, SC, 1982. Resumo. p.117. 
MIRANDA, L.T. A característica "latencia" do milho (Zea mays L.) e suas possibilidades no melhoramento. Piracicaba, ESALQ/USP, 1972.93p. Tese. (Doutoramento)

MONTGOMERY, E.C. Correlation studies of corn. Annual Report, 24th, Nebraska, Agricultural Station, 1911.

SULLIVAN, C.Y.; BENNETT, J.M. \& EASTIN, J.D. Effects on grain sorghum gorwth with root systems only partially in contact available water. In: PROJECT completion Report. Physiological Aspects of water use efficiency. Lincoln, Nebraska, Water Resources Center, University of $\mathrm{Ne}$ braska, 1978.p.44-57.

\& ROSS, W.M. Selecting for drought and heat resistance in grain sorghum. In: MUSSEL, H. \& STAPLES, R. eds. Stress physiology in crop plant. New York, John Wiley and Sons, 1979. p.263-281. 